\title{
Sweat gland bioelectrics differ in cystic fibrosis: a new concept for potential diagnosis and assessment of CFTR function in cystic fibrosis
}

\author{
T Gonska, ${ }^{1,2}$ W Ip, ${ }^{2}$ D Turner, ${ }^{3}$ W S Han, ${ }^{4}$ J Rose, ${ }^{2}$ P Durie, ${ }^{1,2}$ P Quinton ${ }^{4,5}$
}

${ }^{1}$ Department of Paediatrics, University of Toronto, Toronto, Canada; ${ }^{2}$ Physiology and Experimental Medicine, the Research Institute and the Department of Paediatrics, Hospital for Sick Children, Toronto, Canada; ${ }^{3}$ Pediatric Gastroenterology Unit, Shaare Zedek Medical Center, The Hebrew University, Jerusalem, Israel; ${ }^{4}$ Department of

Paediatrics, University of California School of Medicine, San Diego, La Jolla, California, USA; ${ }^{5}$ Division of Biomedical Sciences, University of California, Riverside, California, USA

Correspondence to: Dr P Quinton, Department of Paediatrics, University of California, 9500 Gilman Dr, San Diego, La Jolla, CA 92093-0830, USA; pquinton@ucsd.edu

$\mathrm{PD}$ and $\mathrm{PQ}$ are joint senior authors

Received 16 February 2009 Accepted 18 August 2009 Published Online First 2 September 2009

\begin{abstract}
Background: For nearly 50 years the diagnosis of cystic fibrosis (CF) has depended on measurements of sweat chloride concentration. While the validity of this test is universally accepted, increasing diagnostic challenges and the search for adequate biomarker assays to support curative-orientated clinical drug trials have created a new demand for accurate, reliable and more practical CF tests. A novel concept is proposed that may provide a more efficient real-time method for assessing CFTR function in vivo.

Methods: Cholinergic and $\beta$-adrenergic agonists were iontophoresed to stimulate sweating. The bioelectric potential from stimulated sweat glands (SPD) was measured in vivo using a standard ECG electrode applied to the skin surface. SPD and sweat chloride concentrations were compared in cohorts predicted to express a range of CFTR function as presented by healthy controls $(\mathrm{HC})$, heterozygotes $(\mathrm{Hz})$, pancreatic sufficient (CFPS) and pancreatic insufficient patients with CF (CFPI).
\end{abstract}

Results: The median SPD was hyperpolarised in patients with CF compared with control subjects ( $-47.4 \mathrm{mV}$ vs $-14.5 \mathrm{mV}, \mathrm{p}<0.001)$. In distinguishing between control and CF subjects, SPD (area under receiver operator curve $(A \cup C)=0.997)$ was similar to sweat chloride concentration (AUC $=0.986)$. Sequential cholinergic $/ \beta$ adrenergic sweat stimulation dramatically depolarised the SPD in patients with CF $(p<0.001)$ but had no effect in control subjects $(p=0.6)$ or on the sweat chloride concentration in either group ( $p>0.5)$. Furthermore, the positive SPD response was larger in CFPI than in CFPS subjects $(p=0.04)$.

Conclusion: These results support the concept that skin surface voltages arising from stimulated sweat glands can be exploited to assess expressed CFTR function in vivo and may prove to be a useful diagnostic tool.

Cystic fibrosis (CF) is the most common inherited and life-shortening disorder in the Caucasian population. Defective epithelial chloride channel function due to mutations in the cystic fibrosis transmembrane regulator (CFTR) gene affects secretory and absorptive organs leading to progressive lung disease as a major factor for morbidity and mortality, pancreatic insufficiency, gastrointestinal complications and infertility. ${ }^{1}$ The pathognomic hallmark of high sodium chloride concentrations in sweat from patients with CF formed the basis of the classic sweat test which was developed 50 years ago. ${ }^{2}$

The defect in CFTR chloride channels also results in an altered bioelectrical potential in the CF sweat duct that is approximately tenfold more negative than normal. ${ }^{3}$ Similar though less pronounced observations in airway epithelia led to the introduction of measurements of nasal potential differences (NPD) as an alternative functional diagnostic test for $\mathrm{CF}^{45}$ However, performance of the NPD is technically demanding as well as negatively influenced by inflammation and by exposure to irritants such as cigarette smoke. ${ }^{67}$

Not only are sweat glands one of the richest sources of CFTR chloride channels, but they are arguably the most assessable of affected CF organs. Moreover, in contrast to most other affected organs, sweat glands are not altered by secondary pathologies associated with ductal plugging and inflammation. ${ }^{8}$ We therefore explored the possibility of assessing the difference in the bioelectric properties of sweat glands in vivo by designing a novel diagnostic assay for CF and other diseases ${ }^{9}$ that may offer advantages in economy and simplicity to facilitate diagnoses in less developed settings. We also note the present need for biomarkers to assay new drugs, some of which are now being tested in early phase clinical trials. ${ }^{10-12}$ The need for real-time easily executed outcome measures of the function of CFTR seems likely to increase as multiple clinical sites and larger numbers of subjects enter later phases of trials that require definitive markers of molecular function..$^{13}{ }^{14}$

In this proof of concept study we introduce a novel technique to assess CFTR function in vivo by measuring the transductal voltage of sweat glands at the skin surface (SPD) as a new diagnostic parameter as well as a possible assay for monitoring changing levels of CFTR function in therapeutic trials. We first define the ability of cholinergically-stimulated SPDs to segregate cohorts of healthy controls (HC) and heterozygote carriers $(\mathrm{Hz})$ from pancreatic-sufficient patients with CF (CFPS) and pancreatic-insufficient patients with CF (CFPI). We then present evidence that additional $\beta$-adrenergic stimulation might further improve the diagnostic power of this novel method among cohorts predicted to express a range of CFTR function.

\section{METHODS}

\section{Study participants}

Between July 2006 and December 2007 we prospectively and consecutively ascertained 10 $\mathrm{HC}, 10 \mathrm{~Hz}, 10 \mathrm{CFPS}$ and 8 CFPI. The diagnosis for $\mathrm{CF}$ as well as the exocrine pancreas status was previously established according to current diagnostic criteria. ${ }^{15}{ }^{16}$ Study subjects were selected based on their previous participation in a large diagnostic study, including HC, providing us with 
extended clinical, functional and genetic information. ${ }^{17}$ All study participants signed informed consent and underwent test protocols on at least two occasions.

\section{Sweat stimulation}

Sweat secretion was stimulated using the cholinergic agent pilocarpine 1\% (USP grade, Spectrum, Gardena, USA) applied by iontophoresis for $5 \mathrm{~min}$ to the volar surface of the forearm (Protocol 1). In a second assay at an adjacent skin site we iontophoresed pilocarpine (1\%) as before, but immediately followed this cholinergic stimulation by iontophoresing into this same area a combination of the $\beta$-adrenergic agent isoproterenol $(1 \%)$ and the phosphodiesterase inhibitor aminophylline $(0.8 \%$; USP grade, Spectrum, Gardena, USA and AC\&C, Montreal, Canada) (Protocol 2). Drugs were dissolved in distilled water immediately before use. The $\beta$-adrenergic solution was acidified to $\mathrm{pH} 3.0$ with $\mathrm{HCl}$ to protonate the drugs for maximal transdermal iontophoretic delivery.

To minimise hyperaemia of the skin often seen with the standard quantitative pilocarpine iontophoresis test (OPIT) $\left(50 \mu \mathrm{A} / \mathrm{cm}^{2}\right.$ is recommended as standard operating procedure for the OPIT) and possible trauma to the underlying sweat glands, ${ }^{18}{ }^{19}$ we first determined the iontophoresis current needed for a maximised $\mathrm{SPD}^{20}$ response in 15 additional control subjects (mean age 34.6 years, range 22-60). Maximal SPD and sufficient sweat volumes for chemical analysis were obtained with an applied iontophoresis current of $30 \mu \mathrm{A} / \mathrm{cm}^{2}$, which was therefore used in all subsequent experiments (fig 1).

\section{Sweat chloride concentration}

Sweat chloride concentration was determined by digital chloridometer (LabConco Corporation, Kansas City, Missouri, USA) from sweat collected in the Macroduct collector cup system (Wescor, Logan, USA) with a minimum volume of $20 \mu \mathrm{l}$.

\section{Sweat gland potential difference (SPD) measurements}

After stimulation the skin area was gently cleaned with distilled water and carefully blown dry. Water-saturated mineral oil was applied to the stimulated area to maximise SPDs by minimising transdermal current shunts. An ECG electrode (ConMed, Utica, US) presoaked in $3 \mathrm{M} \mathrm{KCl}$ for $30 \mathrm{~min}$ was taped securely over the prepared area. An angiocath (G22) was inserted subcutaneously by needle puncture. The steel needle was removed and the plastic infusion line was filled with sterile $0.9 \% \mathrm{NaCl}$ solution. The infusion line was then bridged via plastic tubing filled with $3 \mathrm{M} \mathrm{KCl}$ to an $\mathrm{Ag} / \mathrm{AgCl}$ reference electrode. The SPD was measured as the voltage between the ECG electrode and the subcutaneous reference electrode using a voltmeter (Digital Multimeter, ET1039, input impedance $10^{6}$ ohms). The SPD was recorded every minute for 30 min after sweat stimulation. As shown in fig 2, SPD measurements hyperpolarised with time, reaching a plateau 20-30 min after stimulation. An average of the last five minute SPD recordings (minutes 26-30) was used for data analysis.

\section{Electrode asymmetry and drift}

Before and after each test the asymmetry between the reference and the ECG electrode for SPD measurements was assessed by applying the ECG electrode and the infusion line to a $0.9 \%$ $\mathrm{NaCl}$-soaked sponge. Property differences between the ECG and reference electrode, as well as variability between each ECG electrode, apparently resulted in some electrode asymmetry and drift. Data from any electrodes with $> \pm 5.0 \mathrm{mV}$ absolute asymmetry or drift were excluded. The final asymmetry was subtracted from each SPD before further analysis.

\section{Statistical analysis}

Data were summarised as medians with interquartile ranges (IOR, 25th to 75th percentile). Missing data are explained as exclusion of SPD measurements due to asymmetry $> \pm 5.0 \mathrm{mV}$ or unavailable sweat chloride concentration due to insufficient sweat volume. Non-parametric tests (Wilcoxon rank-sum test and Kruskal-Wallis test) were used for comparison between the groups. Analysis for diagnostic test accuracy was performed by determining the area under a receiver-operator characteristic curve (AUC) with its corresponding 95\% confidence interval (CI). An AUC of $>0.7$ was considered as indicative of "fair" discriminative ability, 0.8 as "good" and >0.9 as "excellent". For reliability analysis, two repeated tests in each individual were compared using the $95 \%$ limits of agreement described by Bland and Altman. ${ }^{21}$ For all comparisons, two-tailed tests for a $\mathrm{p}$ value of $<0.05$ were applied to determine significant differences. Graphs and analyses were performed with Prism Version 4.0 (GraphPad Software, 2003) and SPSS Version 16.0, Graduate Student Version, SPSS Inc, 2007).

\section{RESULTS}

The study subjects are described in table 1 . The groups were not sex matched. The mean (SD) ages of the HC (29.7 (8.5) years) and the CFPI (29.9 (4.7) years) cohorts were less than of the $\mathrm{Hz}$
Figure 1 (A) Sweat gland potential difference (SPD) and (B) sweat volume depend on iontophoresis current. Data show medians and IOR (error bars) of measurements on number $(n)$ of healthy controls. Each of 15 healthy individuals underwent 2-6 sweat tests using cholinergic stimulation by iontophoresis with current ranging from 2.5 to $50 \mu \mathrm{A} / \mathrm{cm}^{2}$. The dose-effect curves were fitted ?using non-linear regression based on a simple receptor-ligand kinetic $(\mathrm{Y}=\mathrm{YO}+$ $(Y \max -\mathrm{YO}) \times \mathrm{X} /(\mathrm{K}+\mathrm{X}))$. Since larger currents did not significantly alter SPD or sweat volumes, an iontophoresis current of $30 \mu \mathrm{A} / \mathrm{cm}^{2}$ was considered optimal for SPD results and sweat volume for sweat chloride analysis $(>20 \mu \mathrm{l})$.
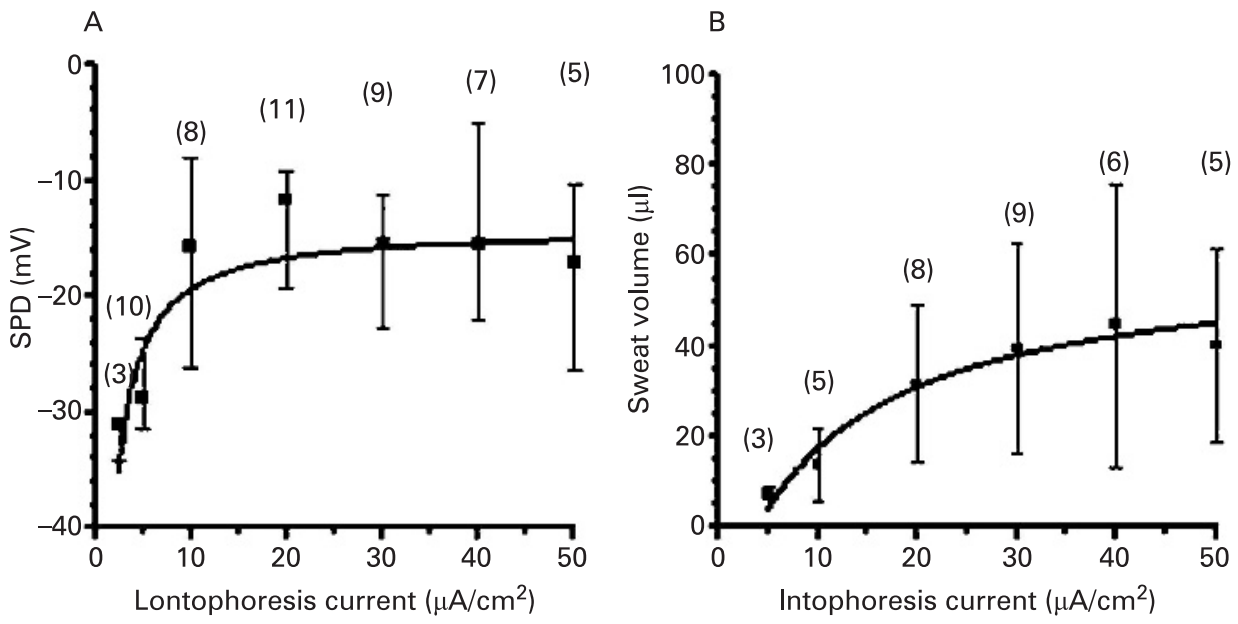


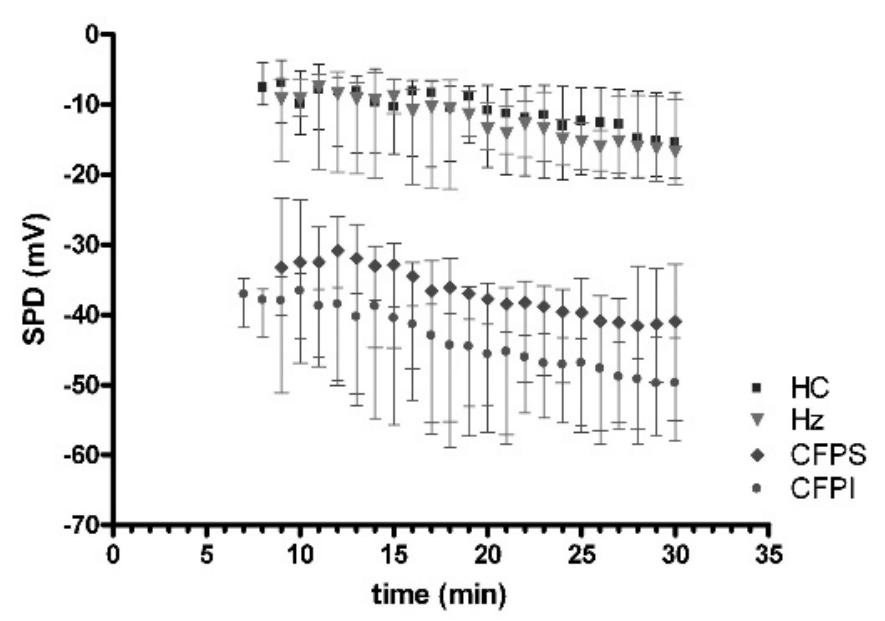

Figure 2 Summary of recordings of sweat gland potential difference (SPD). SPD hyperpolarised over time, but a plateau was achieved 20-30 min after stimulation in all subjects. The data acquired during the last 5 min of the 30 min after iontophoresis of pilocarpine were therefore averaged for each subject and used as a single SPD value for that subject in further analysis. Each data point is the median and IQR of the SPD at the time indicated in minutes following cholinergic sweat stimulation for each of the four groups: $\mathrm{HC}$, healthy control $(n=9) ; \mathrm{Hz}$, heterozygotes $(n=10)$; CFPS, patients with pancreatic sufficient cystic fibrosis $(n=10)$; CFPI, patients with pancreatic insufficient cystic fibrosis $(n=8)$.

(41.3 (13.9) years) and CFPS (41.9 (9.6) years) cohorts. However, there was no statistically significant age difference between the combined groups of $\mathrm{HC} / \mathrm{Hz}$ and CFPS/CFPI $(p=0.8)$.

\section{SPD in patients with CF and healthy controls}

In Protocol 1, sweat was stimulated only with the cholinergic drug pilocarpine (1\%). Considering all $\mathrm{HC}$ and $\mathrm{Hz}$ individuals as a control group and all CFPS and CFPI patients as a CF group, sweat gland SPDs were significantly hyperpolarised in the CF group compared with the control group $(-47.4 \mathrm{mV}$ vs $-14.5 \mathrm{mV}, \mathrm{p}<0.001)$. In an area immediately adjacent to the SPD measurement site, sweat was collected for determination of chloride concentration. The sweat chloride concentration was also significantly higher in the CF group than in the control group ( $98.5 \mathrm{mmol} / \mathrm{l}$ vs $21.5 \mathrm{mmol} / \mathrm{l}, \mathrm{p}<0.001)$. Table 2 shows the SPD and sweat chloride concentration data for all groups.

\section{SPD compared with sweat chloride concentration}

The power of the SPD assay to distinguish between different groups was comparable to that of the sweat chloride assay (table 2, fig 3). Both assays were plotted in relation to each other, revealing good correlation of SPD to sweat chloride concentration obtained simultaneously ( $r=-0.7,95 \%$ CI -0.84 to $-0.48, p<0.001$; fig 4). Furthermore, using ROC as an analytical tool, SPD was found to have a similar accuracy to sweat chloride concentration in identifying patients with CF, with equal excellent AUC of 0.997 (95\% CI 0.988 to 1.007) for SPD and 0.986 (95\% CI 0.958 to 1.014$)$ for sweat chloride concentration (fig 4).

Neither sweat chloride concentration nor cholinergicallystimulated SPD resulted in a significant difference between HC and $\mathrm{Hz}$ ( $p=0.7$ and $\mathrm{p}=0.6$, respectively) or between CFPS and CFPI ( $p=0.2$ and $p=0.5$, respectively).

For 31 of 37 study subjects, repeated SPD and sweat chloride measurements were available (fig 4). Analysing the differences between test and retest results by the Bland-Altman method, ${ }^{21}$ sweat chloride concentration showed better repeatability for most patients with CF (values $>40 \mathrm{mmol} / \mathrm{l}$ ), whereas equal but higher variability was seen for SPD throughout the whole functional spectrum. However, there was no fixed or proportional bias for either test and the 95\% confidence limits of agreement were comparable. Despite the wider difference between two tests seen for SPD in some study subjects, the second SPD test did not alter the classification of individuals in their subgroup (table 2).

Table 1 Summary of study subjects

\begin{tabular}{|c|c|c|c|c|c|c|c|c|c|}
\hline ID & Category & Sex & Age & Genotype & ID & Category & Sex & Age & Genotype \\
\hline 1 & $\mathrm{HC}$ & $\mathrm{F}$ & 49 & $+/+$ & 21 & CFPS & $M$ & 46 & deltaF508/P67L \\
\hline 2 & $\mathrm{HC}$ & $\mathrm{F}$ & 39 & $+/+$ & 22 & CFPS & $\mathrm{F}$ & 41 & deltaF508/R117C \\
\hline 3 & $\mathrm{HC}$ & $\mathrm{M}$ & 32 & $+/+$ & 23 & CFPS & $\mathrm{F}$ & 57 & G542X/D1152H \\
\hline 4 & $\mathrm{HC}$ & $\mathrm{M}$ & 23 & $+/+$ & 24 & CFPS & M & 34 & deltaF508/M1101K \\
\hline 5 & $\mathrm{HC}$ & $\mathrm{F}$ & 28 & $+/+$ & 25 & CFPS & $\mathrm{F}$ & 29 & deltaF508/L1335P \\
\hline 6 & $\mathrm{HC}$ & $\mathrm{M}$ & 26 & $+/+$ & 26 & CFPS & $\mathrm{F}$ & 48 & deltaF508/+ \\
\hline 7 & $\mathrm{HC}$ & $\mathrm{M}$ & 26 & R750/+ & 27 & CFPS & $\mathrm{M}$ & 26 & deltaF508/R117H \\
\hline 8 & $\mathrm{HC}$ & $\mathrm{M}$ & 30 & $+/+$ & 28 & CFPS & $\mathrm{M}$ & 44 & deltaF508/3272_26A $>G$ \\
\hline 9 & $\mathrm{HC}$ & $\mathrm{M}$ & 22 & $+/+$ & 29 & CFPS & $\mathrm{M}$ & 46 & deltaF508/R117H 5T \\
\hline 10 & $\mathrm{HC}$ & $\mathrm{M}$ & 22 & $+/+$ & 30 & CFPS & $\mathrm{M}$ & 48 & $\mathrm{R} 347 \mathrm{P} / 2753-2 \mathrm{~A}>\mathrm{G}$ \\
\hline 11 & $\mathrm{~Hz}$ & $\mathrm{~F}$ & 26 & deltaF508/+ & 31 & CFPI & M & 29 & deltaF508/deltaF508 \\
\hline 12 & $\mathrm{~Hz}$ & $\mathrm{~F}$ & 54 & deltaF508/+ & 32 & CFPI & $\mathrm{M}$ & 29 & deltaF508/2194inA \\
\hline 13 & $\mathrm{~Hz}$ & $\mathrm{~F}$ & 24 & deltaF508/+ & 33 & CFPI & $\mathrm{F}$ & 40 & $\mathrm{G} 551 \mathrm{D} / 621+1 \mathrm{G}>\mathrm{T}$ \\
\hline 14 & $\mathrm{~Hz}$ & $\mathrm{~F}$ & 33 & deltaF508/+ & 34 & CFPI & $\mathrm{M}$ & 33 & deltaF508/deltaF508 \\
\hline 15 & $\mathrm{~Hz}$ & $\mathrm{M}$ & 25 & deltaF508/+ & 35 & CFPI & $\mathrm{M}$ & 27 & deltaF508/deltaF508 \\
\hline 16 & $\mathrm{~Hz}$ & $\mathrm{~F}$ & 37 & deltaF508/+ & 36 & CFPI & M & 25 & deltaF508/deltaF508 \\
\hline 17 & $\mathrm{~Hz}$ & $\mathrm{~F}$ & 49 & deltaF508/+ & 37 & CFPI & $\mathrm{M}$ & 27 & deltaF508/deltaF508 \\
\hline 18 & $\mathrm{~Hz}$ & $\mathrm{M}$ & 49 & deltaF508/+ & 38 & CFPI & $\mathrm{M}$ & 29 & deltaF508/deltaF508 \\
\hline 19 & $\mathrm{~Hz}$ & $\mathrm{~F}$ & 55 & deltaF508/+ & & & & & \\
\hline 20 & $\mathrm{~Hz}$ & $\mathrm{M}$ & 61 & deltaF508/+ & & & & & \\
\hline
\end{tabular}

CFPI, pancreatic-insufficient CF patients; CFPS, pancreatic-sufficient CF patients; HC, healthy controls; Hz, heterozygotes.

CFTR gene sequencing was performed for all subjects. ${ }^{22}{ }^{23}$ 
Table 2 Comparison of SPD and sweat chloride concentration for all groups

\begin{tabular}{|c|c|c|c|c|c|c|c|c|}
\hline & \multicolumn{4}{|c|}{ SPD-cholinergic (Protocol 1) } & \multicolumn{2}{|c|}{$\Delta$ SPDchol/B-adren (Protocol 2) } & \multicolumn{2}{|c|}{ Sweat chloride-cholinergic } \\
\hline & (1) Test & p Value* & (2) Test & p Value* & & p Value* & & p Value* \\
\hline \multirow[t]{2}{*}{$\mathrm{HC}$} & -14.4 & 0.6 & -17.6 & 0.5 & -0.1 & 0.5 & 23.5 & 0.7 \\
\hline & (9) & & (9) & & (8) & & (10) & \\
\hline \multirow[t]{2}{*}{$\mathrm{Hz}$} & -16.0 & & -16.8 & & 1.8 & & 18 & \\
\hline & $\begin{array}{l}-20.5 \text { to }-6.7 \\
\text { (10) }\end{array}$ & & $\begin{array}{l}-19.6 \text { to }-8.3 \\
\text { (10) }\end{array}$ & & $\begin{array}{l}-0.6 \text { to } 6.4 \\
\text { (7) }\end{array}$ & & $\begin{array}{l}13.5 \text { to } 28.5 \\
\text { (10) }\end{array}$ & \\
\hline CFPS & $\begin{array}{l}-55.9 \text { to }-37.4 \\
\text { (10) }\end{array}$ & & $\begin{array}{l}-51.2 \text { to }-28.5 \\
\text { (5) }\end{array}$ & & $\begin{array}{l}12.3 \text { to } 19.8 \\
\text { (8) }\end{array}$ & & $\begin{array}{l}68 \text { to } 103.5 \\
\text { (8) }\end{array}$ & \\
\hline \multirow[t]{2}{*}{ CFPI } & -48.8 & & -55.2 & & 24.0 & & 104 & \\
\hline & $\begin{array}{l}-56.8 \text { to }-43.5 \\
\text { (8) }\end{array}$ & & $\begin{array}{l}-63.8 \text { to }-43.3 \\
\text { (7) }\end{array}$ & & $\begin{array}{l}19.6 \text { to } 29.1 \\
\text { (7) }\end{array}$ & & $\begin{array}{l}92.5 \text { to } 119.5 \\
\text { (8) }\end{array}$ & \\
\hline $\mathrm{HC}+\mathrm{Hz}$ & -14.5 & $<0.001$ & -17.6 & $<0.001$ & 1.5 & $<0.001$ & 21.5 & $<0.001$ \\
\hline
\end{tabular}

All data are given as medians, interquartile range (IOR) and number of subjects (n).

CFPI, pancreatic-insufficient CF patients; CFPS, pancreatic-sufficient CF patients; $\mathrm{HC}$, healthy controls; $\mathrm{Hz}$, heterozygotes; SPD, sweat gland potential difference; $\triangle \mathrm{SPD}$ chol/ $\beta$-adren, sweat gland potential difference recorded after cholinergic followed immediately by $\beta$-adrenergic stimulation minus SPD recorded after cholinergic stimulation alone.

*Wilcoxon rank sum test.

\section{SPD after sequential cholinergic/价adrenergic stimulation}

In an attempt to directly activate CFTR chloride channels and improve the segregation of subgroups, we modified the sweat stimulation protocol using sequential stimulation with cholinergic followed by $\beta$-adrenergic agonists (Protocol 2). Interestingly and unexpectedly, sequential stimulation with cholinergic and then $\beta$-adrenergic agonists had no effect on median SPD in $\mathrm{HC}$ and $\mathrm{Hz}$ as control group $(\mathrm{p}=0.6)$, but significantly depolarised the SPD of all patients with CF $(p<0.001$, fig 5$)$. Sweat chloride concentration did not change in either group following sequential stimulation $(\mathrm{HC} / \mathrm{Hz}$ : $p=0.95$; CFPS/CFPI: $p=0.94$; fig 5 )

Calculating the difference of the SPD response between cholinergic and sequential cholinergic/ $\beta$-adrenergic stimulation $\left(\Delta \mathrm{SPD}_{\text {chol }} / \beta_{\text {-adren }}\right)$, we found a statistically significant difference between controls and patients with CF $(p<0.001)$. After sequential stimulation neither the SPD of $\mathrm{HC}$ nor $\mathrm{Hz}$ subjects changed significantly, whereas the SPD of CFPS and CFPI patients was significantly depolarised with both agonists. Thus, subgroup analysis showed no statistical difference between the response of $\mathrm{HC}$ and $\mathrm{Hz} \quad(p=0.5)$. However, SPD was significantly more positive in CFPI patients following sequential cholinergic/ $\beta$-adrenergic sweat stimulation than in CFPS patients, so that $\Delta \mathrm{SPD}_{\text {chol } /} \beta_{\text {-adren }}$ between CFPS and CFPI was significantly different ( $p=0.04$; table 2 , fig 5$)$.

\section{Adverse events}

No severe systemic or local adverse events were observed. About $20 \%$ of the study subjects showed very mild transient reddening of the skin following iontophoresis. One subject had a moderate skin reaction (reddening) which resolved after $30 \mathrm{~min}$.

\section{DISCUSSION}

\section{A novel sweat test}

For over 50 years the gold standard test in CF has been the OPIT which involves a multistep procedure of (1) in vivo stimulation of the sweat glands; (2) collection of the sweat secretion; and (3) laboratory determination of the sweat chloride concentration. ${ }^{24}$ We have now explored the increased
Figure 3 Comparison of sweat gland potential difference (SPD) and sweat chloride concentration $\left(\left[\mathrm{Cl}^{-}\right]\right)$ measurements. Each subject underwent simultaneous SPD and sweat chloride measurements; (n) number of subjects. Scatter plots show $(A)$ the average of SPDs recorded during the last 5 min after stimulation and $(B)$ the sweat chloride concentration for each individual in each group. SPD and sweat chloride concentrations show significantly different medians between the control and patient groups $(\mathrm{p}<0.001)$. $\mathrm{HC}$, healthy control; $\mathrm{Hz}$, heterozygotes; CFPS, patients with pancreatic-sufficient cystic fibrosis; CFPI, patients with pancreatic-insufficient cystic fibrosis.
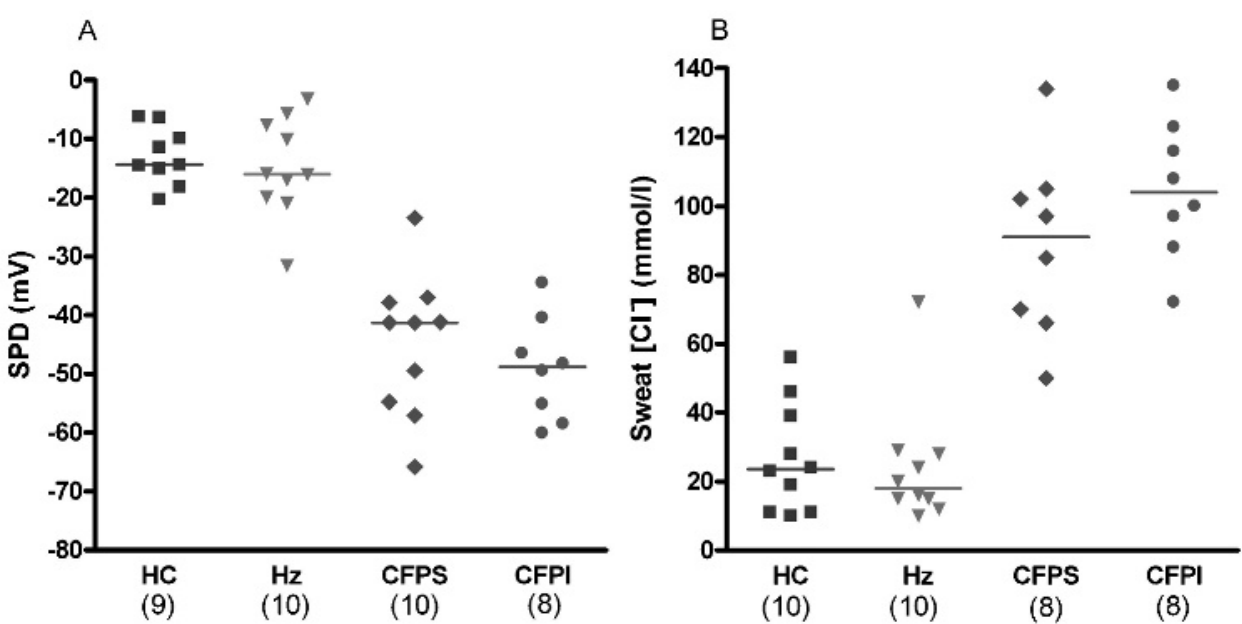
Figure 4 Characteristics of sweat gland potential difference (SPD) and sweat chloride concentration $\left(\left[\mathrm{Cl}^{-}\right]\right)$tests following cholinergic sweat stimulation. Receiver operator curves, which plot the sensitivity (true positive rate) versus the 1-specificity (false positive rate), were used as an analytical tool to test for the ability of SPD compared with sweat chloride concentration to distinguish between control and disease. (A) Area under the curve (AUC) is $0.997(95 \% \mathrm{Cl}$ 0.988 to 1.007$)$ for SPD and $0.986(95 \%$ Cl 0.958 to 1.014$)$ for sweat chloride concentration. (B) SPD showed good correlation with the simultaneously performed sweat chloride concentration measurement $(r=-0.7,95 \% \mathrm{Cl}-0.84$ to $-0.48, p<0.001)$. Test and retest analysis using the Bland-Altman method of differences showed no fixed bias between two tests for (C) SPD $(2.9 \mathrm{mV}$, $\mathrm{p}=0.4$ ) or (D) sweat chloride

concentration $(2.2 \mathrm{mmol} / \mathrm{l}, \mathrm{p}=0.8)$ and no proportional bias between two tests for SPD (Spearman $r=0.05, p=0.8$ ) or sweat chloride concentration (Spearman $r=0.1, p=0.6)$. The $95 \%$ confidence limits were -18.6 to $24.5 \mathrm{mV}$ for SPD and -16 to $20.3 \mathrm{mmol} / \mathrm{l}$ for sweat chloride concentration.
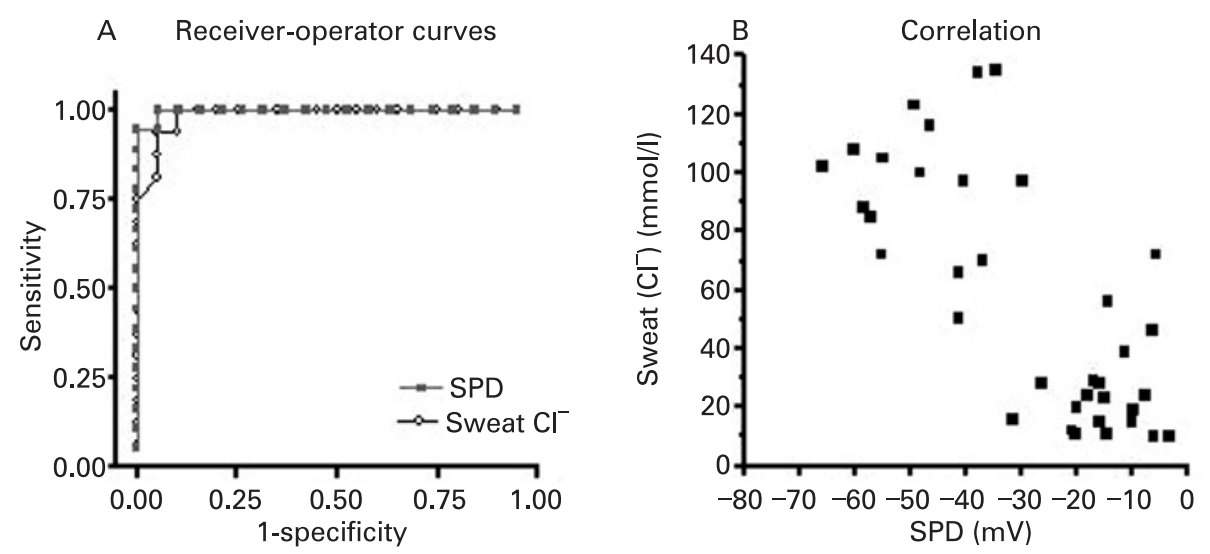

Bland Altman plots
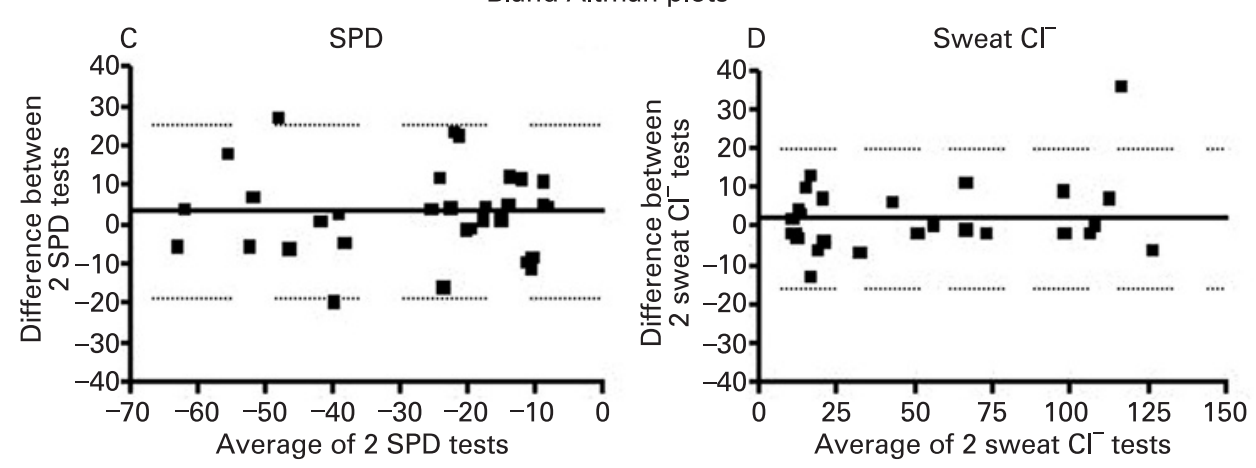

negative bioelectric potential arising directly from the basic CF defect to introduce a proof of concept for a new simple practical real-time assay of sweat gland function which appears to be as accurate as the traditional OPIT and, perhaps, with further technical improvement in electrode design, may prove to be more reproducible and sensitive.

The bioelectrical properties of isolated sweat ducts have been extensively studied. ${ }^{3}$ 8 Moreover, in vivo measurements in sweat droplets from single glands on the skin showed that the electrical potential of the sweat ducts can be recorded at the gland orifice on the skin surface. ${ }^{20}{ }^{25}$ We therefore surmised that placing an ECG electrode over an area of stimulated skin would measure the average of the electrical potentials from ducts of all secreting sweat glands $(>1000)$ under the electrode. This skin potential could, in turn, serve as a real-time diagnostic measure without further procedures such as chemically analysing sweat. Moreover, since the electrical potential from the sweat duct directly reflects the function of CFTR, this technique might assess the impact of drugs administered in clinical trials for correcting the defect in CFTR chloride conductance.

\section{SPD as a biomarker assay for CF}

In order to establish the usefulness of the sweat gland SPD for assessing CFTR function, we measured phenotypically and genotypically well-characterised cohorts of $\mathrm{HC}, \mathrm{Hz}$, patients with CFPS and patients with CFPI. We anticipate that these cohorts should serve as surrogates for distinct levels of CFTR function that might be induced by corrective therapies. We demonstrated that the SPD (fig 3) was significantly more negative in patients with CF than in control subjects $(-47.4 \mathrm{mV}$ vs $-14.5 \mathrm{mV}$; table 2$)$, which corresponded well to previous results on single glands $(-66 \mathrm{mV}$ vs $-29 \mathrm{mV}){ }^{25}$

In our preselected cross-sectional cohort, SPD appeared to be as effective as the classic OPIT sweat chloride concentration in segregating the different subgroups (fig 3), giving equal diagnostic accuracy to identifying patients with CF (fig 4). This finding must now be confirmed in a larger unselected and more representative population in a future study. The high correlation between SPD and sweat chloride concentration indicates that both methods measure phenomena related to the same defect (CFTR) in sweat glands. Repeatability analysis showed larger variability in the control group for sweat chloride concentration and an overall larger but similar variability in the controls and CF group for SPD measurements. However, the absence of any bias in the Bland-Altman analysis, as well as comparable limits of agreement, suggest acceptable repeatability for SPD measurements. The larger variability in SPD measurements observed in some individuals may be due, at least partly, to reduced sensitivity caused by electrode asymmetries (discussed later) and should be reduced with improvements in electrode design.

Previous studies in a larger cohort showed a slight elevation in mean sweat chloride concentration in $\mathrm{Hz}$ compared with $\mathrm{HC}$ and a statistically lower mean sweat chloride concentration in patients with CFPS compared with those with CFPI, although the overlap between the groups precluded segregation of individuals. ${ }^{26}$ An assay that provides an increased ability to detect differences between subgroups, particular between CFPS and CFPI, based on small increments in CFTR function, may therefore have significant value for assessing degrees of correction achieved by drug therapy to modify mutant CFTR function. However, neither sweat chloride concentration nor cholinergically-induced SPD testing distinguished between $\mathrm{HC}$ and $\mathrm{Hz}$ or between CFPS and CFPI in this small cohort (fig 3, table 1).

Sequential cholinergic/ $\beta$-adrenergic sweat stimulations promise to improve diagnostic performance of SPD

We reasoned that maximally stimulating CFTR in all groups might enhance differences in SPD responses between the 
Figure 5 Sweat gland potential difference (SPD) and sweat chloride concentration $\left(\left[\mathrm{Cl}^{-}\right]\right)$following sequential cholinergic/ $\beta$-adrenergic sweat stimulation. Each subject underwent contemporaneous sweat chloride and two SPD measurements. One SPD was measured after cholinergic (chol) alone and another SPD after sequential cholinergic/ $\beta$-adrenergic ( $\beta$-adren) stimulation (see Methods section). Connecting lines indicate sequential measurements of SPD and sweat chloride concentration in the same subject.

(A) SPD values remained relatively constant for healthy controls and heterozygotes, but depolarised markedly in all CF patients. (B) The sweat chloride values were not detectably altered in any group. Box plots show medians and IQR of the difference between chol and $\beta$-adreninduced SPD responses $\left(\triangle S P D_{\text {chol }} \beta_{\text {-adren }}\right)$. $(C, D)$ Not only are the $\triangle S P D_{\text {chol }} \beta_{\text {-adren }}$ changes significantly different for patients compared with control groups $\left({ }^{*} p<0.001\right)$, but the amount of depolarisation is significantly less in CFPS patients than in CFPI patients $\left({ }^{* *} p=0.04\right)$. HC, healthy control; $\mathrm{Hz}$, heterozygotes; CFPS, patients with pancreatic-sufficient cystic fibrosis; CFPI, patients with pancreatic-insufficient cystic fibrosis.

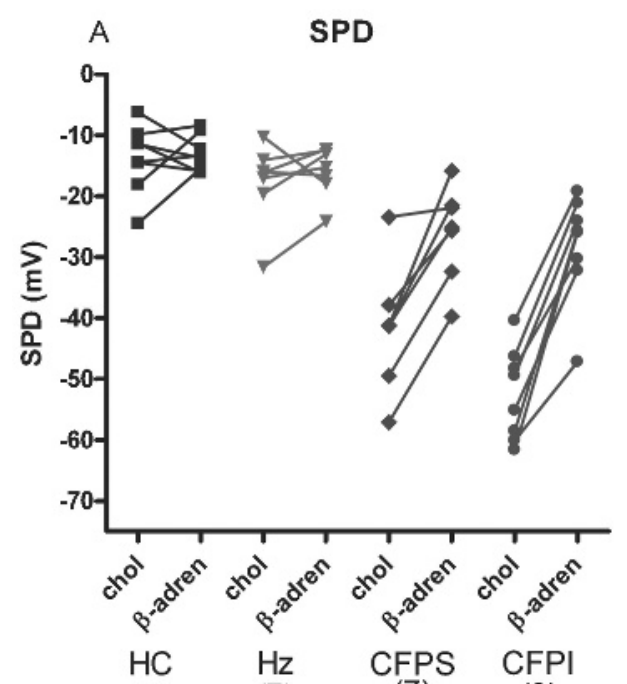

(8)

(7)

(7)

(8)

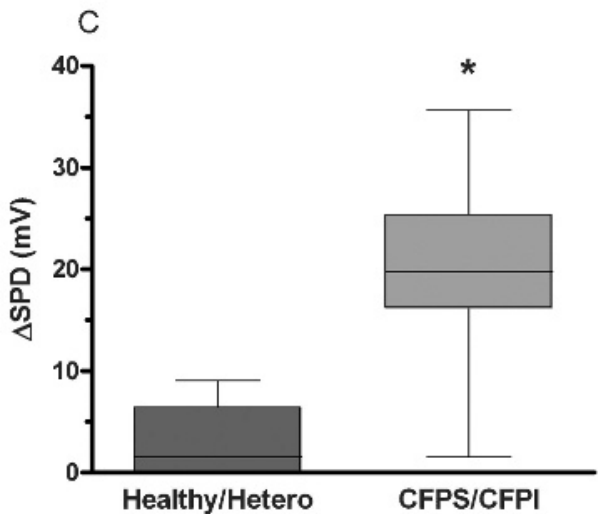

(15)

(15)

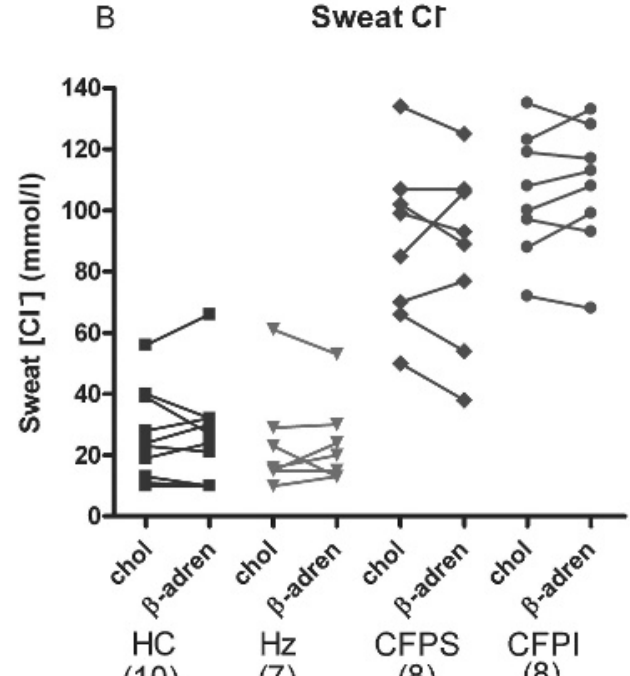

(10)

(7)

(8)

(8)

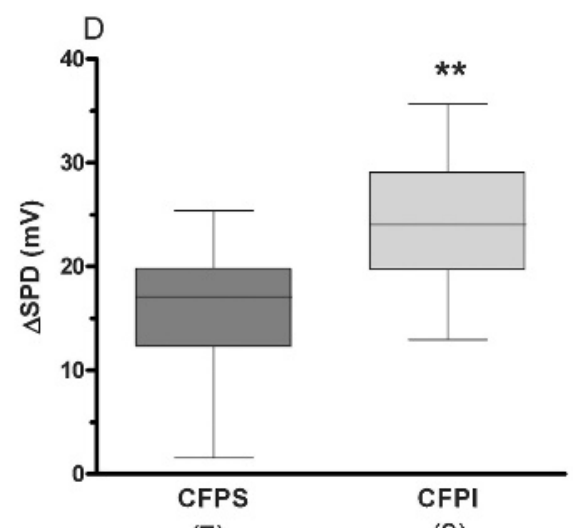

(7) subgroups where theoretically, at least, CFTR function is twofold greater in $\mathrm{HC}$ than in $\mathrm{Hz}$ subjects and is probably greater in patients with CFPS than in those with CFPI. Following cholinergic sweat stimulation we iontophoresed a mixture of the $\beta$-adrenergic agonists isoproterenol and aminophylline to enhance cAMP levels and maximally stimulate CFTR activity. We expected maximal effects, if any, in control subjects where CFTR is highly expressed. However, stimulation with $\beta$-agonists did not change the average SPD in either $\mathrm{HC}$ or $\mathrm{Hz}$ subjects but, surprisingly, increased (depolarised) the SPD response in all patients with CF, with the greatest effect in those with CFPI (fig 5). The differences between the SPD response following cholinergic sweat stimulation alone and sequential cholinergic/ $\beta$-adrenergic stimulation $\left(\Delta \mathrm{SPD}_{\text {chol }} \beta_{\text {-adren }}\right)$ was not only significantly different between controls and patients with CF $(p<0.001)$, but also between CFPS from CFPI $(p=0.04)$. In fact, the $\triangle \mathrm{SPD}_{\text {chol }} \beta_{\text {-adren }}$ linearly correlated with the measured baseline SPD ( $r=-0.8,95 \% \mathrm{CI}-0.92$ to $-0.69, \mathrm{p}<0.001)$, suggesting that the $\beta$-adrenergic SPD response is directly related to the degree of CFTR dysfunction The $\Delta \mathrm{SPD}_{\text {choll }} \beta_{\text {-adren }}$ might support further segregation between different CF subgroups in the future. A range of $\Delta S \mathrm{SPD}_{\text {chol }} \beta_{\text {-adren }}$ in the CFPS and CFPI subjects is anticipated because different mutations in the CFTR gene express varying degrees of residual CFTR function.

Why does $\beta$-adrenergic sweat stimulation depolarise SPD in CF? While the $\beta$-adrenergically unaltered SPD response in controls may be explained by the presence of constitutively fully-activated
CFTR in these glands, ${ }^{8}$ the dramatic depolarisation in patients with CF seems puzzling. We expected that less (or no) CFTR expression in patients with CF would result in smaller (or no) changes in SPD, not larger. Two scenarios might possibly explain these results. First, $\beta$-adrenergic activation may stimulate or insert additional mutant CFTR in the membrane which would shunt the electrogenic potentials in these ducts, thus leading to a more positive "normalised" SPD. ${ }^{27}$ However, increased CFTR conductance should have decreased sweat chloride concentration, which was not observed. Moreover, $\beta$-adrenergic stimulation is not known to increase the expression of $\Delta$ F508 mutations.

Second, and perhaps more likely, $\beta$-adrenergic stimulation appears to stimulate acid secretion by the sweat duct ${ }^{28}{ }^{29}$ which should depolarise the lumen and hence the SPD. The presence of a small chloride conductance, as expected for some mutations, would partially shunt this potential, as seen in patients with CFPS, and reduce-but not abolish - the depolarising effect of proton secretion.

\section{Technical challenges of the SPD sweat test}

Using a simple ECG set-up for SPD recordings, we have achieved excellent statistical separations. However, artefacts introduced by asymmetries and voltage drifts between the measuring and reference electrodes ( $> \pm 5 \mathrm{mV}$ in up to $35 \%$ of experiments) may account for some of the intra- and inter-individual variability. These errors may have been caused by (1) changes in the composition of the gel of the measuring ECG electrode due to exposure to different volumes and salt concentrations in 
secreted sweat; ${ }^{30}$ and (2) use of unmatched electrodes, ECG gel vs agar $\mathrm{Ag} / \mathrm{AgCl}$. Improvements in the design of the electrode are expected to minimise these artefacts and increase the accuracy of the SPD recording. Furthermore, to obviate the need for subcutaneous needles in paediatric subjects, other means of establishing an electrical reference with the serosal fluid such as a minor skin abrasion may prove preferable.

In summary, the findings in this paper show, for the first proof of concept, that cholinergically-stimulated SPDs can be used as an immediate, simple and accurate method to assess CFTR function in vivo. Including a second protocol to compare cholinergically-stimulated SPDs with combined cholinergically and $\beta$-adrenergically-stimulated SPDs increased the power of the test to detect smaller differences in CFTR function, notably between subjects with CFPS and CFPI. These observations therefore justify further development of the SPD as an outcome parameter in clinical trials and as a diagnostic test for CF.

Acknowledgements: The authors thank all volunteers and especially CF patients for participating in the study; Jennifer Pike, Dr Elizabeth Tullis, Susan Carpenter, Louise Taylor, Felix Ratjen, Douglas Conrad and Mark Pian for patient referral; Dr Christine Bear for her support and advice on test development; Lynda Ellis and Sheelagh Martin for their assistance with the clinical data; Julie Gibson for advice on communication with regulatory authorities; and special thanks to Annie Dupuis for assistance with the statistical analysis.

Funding: The study was funded by the Canadian Cystic Fibrosis Foundation as part of the BREATHE project, a Fellowship award to TG, the US Cystic Fibrosis Foundation to $\mathrm{PQ}$ and PD and the Nancy Olmsted Trust to PQ.

Competing interests: None.

Ethics approval: The ethical boards of the Hospital for Sick Children and St. Michael's Hospital in Toronto, Canada, as well as Health Canada, approved the study.

Provenance and peer review: Not commissioned; externally peer reviewed.

\section{REFERENCES}

1. Welsh MJ, Ramsey BW, Accurso F, et al. Cystic fibrosis. In: Scriver CR, Beaudet AL, Sly WS, Walle D, eds. The metabolic and molecular bases of inherited disease. New York: McGraw-Hill, 2001:5121-88.

2. Gibson LE, Cooke RE. A test for concentration of electrolytes in sweat in cystic fibrosis of the pancreas utilizing pilocarpine by iontophoresis. Pediatrics 1959;23:545-9.

3. Quinton PM. Chloride impermeability in cystic fibrosis. Nature 1983;301:421-2.

4. Knowles M, Gatzy J, Boucher R. Increased bioelectric potential difference across respiratory epithelia in cystic fibrosis. N Engl J Med 1981;305:1489-95.

5. Knowles MR, Paradiso AM, Boucher RC. In vivo nasal potential difference: techniques and protocols for assessing efficacy of gene transfer in cystic fibrosis. Hum Gene Ther 1995;6:445-55.

6. Ahrens RC, Standaert TA, Launspach J, et al. Use of nasal potential difference and sweat chloride as outcome measures in multicenter clinical trials in subjects with cystic fibrosis. Pediatr Pulmonol 2002;33:142-50.
7. Cantin AM, Hanrahan JW, Bilodeau G, et al. Cystic fibrosis transmembrane conductance regulator function is suppressed in cigarette smokers. Am J Respir Crit Care Med 2006;173:1139-44.

8. Quinton PM. Cystic fibrosis: lessons from the sweat gland. Physiology (Bethesda) 2007;22:212-25.

9. Reddy MM, Wang XF, Gottschalk M, et al. Normal CFTR activity and reversed skin potentials in pseudohypoaldosteronism. J Membr Biol 2005;203:151-9.

10. Moss RB, Milla C, Colombo J, et al. Repeated aerosolized AAV-CFTR for treatment of cystic fibrosis: a randomized placebo-controlled phase 2B trial. Hum Gene Ther 2007:18:726-32.

11. Kerem E, Hirawat S, Armoni S, et al. Effectiveness of PTC124 treatment of cystic fibrosis caused by nonsense mutations: a prospective phase II trial. Lancet 2008;372:719-27.

12. Clancy JP, Rowe SM, Bebok Z, et al. No detectable improvements in cystic fibrosis transmembrane conductance regulator by nasal aminoglycosides in patients with cystic fibrosis with stop mutations. Am J Respir Cell Mol Biol 2007;37:57-66.

13. Rowe SM, Accurso F, Clancy JP. Detection of cystic fibrosis transmembrane conductance regulator activity in early-phase clinical trials. Proc Am Thorac Soc 2007; 4:387-98.

14. Mayer-Hamblett N, Ramsey BW, Kronmal RA. Advancing outcome measures for the new era of drug development in cystic fibrosis. Proc Am Thorac Soc 2007:4:370-7.

15. Farrell PM, Rosenstein BJ, White TB, et al. Guidelines for diagnosis of cystic fibrosis in newborns through older adults: Cystic Fibrosis Foundation Consensus Report. $J$ Pediatr 2008;153:S4-14.

16. Kristidis P, Bozon D, Corey $M$, et al. Genetic determination of exocrine pancreatic function in cystic fibrosis. Am J Hum Genet 1992:50:1178-84.

17. Dupuis A, Tullis E, Ellis L, et al. Developing a cystic fibrosis spectrum score (abstract). Pediatr Pulmonol Supp/ 2008;31:280.

18. Rattenbury JM, Worthy E. Is the sweat test safe? Some instances of burns received during pilocarpine iontophoresis. Ann Clin Biochem 1996;33:456-8.

19. LeGrys VA, Retsch-Bogart GZ. Urticaria associated with the pilocarpine iontophoresis sweat test. Pediatr Pulmonol 1997;24:296-7.

20. Bijman J, Quinton PM. Influence of abnormal $\mathrm{Cl}^{-}$impermeability on sweating in cystic fibrosis. Am J Physiol 1984;247:C3-9.

21. Bland JM, Altman DG. Statistical methods for assessing agreement between two methods of clinical measurement. Lancet 1986;1:307-10.

22. Chu CS, Trapnell BC, Curristin S, et al. Genetic basis of variable exon 9 skipping in cystic fibrosis transmembrane conductance regulator mRNA. Nat Genet 1993;3:151-6.

23. Zielenski J, Aznarez I, Onay T, et al. CFTR mutation detection by multiplex heteroduplex (mHET) analysis on MDE gel. Methods Mol Med 2002;70:3-19.

24. LeGrys VA, Rosenstein BJ, Doumas BT, et al. Sweat testing: sample collection and quantitative analysis; approved guideline. 2nd edn. Clinical Laboratory Standards Institute (formerly NCCLS). Document C34-a2.pdf, June 2007.

25. Quinton P, Bijman J. Higher bioelectric potentials due to decreased chloride absorption in the sweat glands of patients with cystic fibrosis. N Engl J Med 1983;308:1185-9.

26. Wilschanski $\mathbf{M}$, Dupuis A, Ellis L, et al. Mutations in the cystic fibrosis transmembrane regulator gene and in vivo transepithelial potentials. Am J Respir Crit Care Med 2006;174:787-94.

27. Carvalho-Oliveira I, Efthymiadou A, Malhó R, et al. CFTR localization in native airway cells and cell lines expressing wild-type or F508del-CFTR by a panel of different antibodies. J Histochem Cytochem 2004:52:193-203.

28. Reddy MM, Quinton PM. Chloride and bicarbonate transport in the human sweat duct. In: Mastella G, Quinton PM, eds. Cellular and molecular basis of cystic fibrosis. San Francisco: San Francisco Press, 1988:125-32.

29. Quinton P, Reddy MM. Cl-conductance and acid secretion in the human sweat duct. Ann NY Acad Sci 1989:574:438-46.

30. Neher E. Correction for liquid junction potentials in patch clamp experiments. Methods Enyzmol 1992;207:123-31. 